\title{
Multiexciton Generation in Seeded Nanorods
}

\author{
Hagai Eshet, ${ }^{* \dagger}{ }^{\dagger}$ Roi Baer, ${ }^{*}{ }^{* \dagger}$ Daniel Neuhauser, ${ }^{*}, \mathbb{\Phi}$ and Eran Rabani ${ }^{*}{ }^{\dagger}$ \\ ${ }^{\dagger}$ School of Chemistry, The Sackler Faculty of Exact Sciences, Tel Aviv University, Tel Aviv 69978, Israel \\ ${ }^{\ddagger}$ Fritz Haber Center for Molecular Dynamics, Institute of Chemistry, The Hebrew University of Jerusalem, Jerusalem 91904, Israel \\ ${ }^{\mathbb{I I}}$ Department of Chemistry, University of California at Los Angeles, Los Angeles, California 90095, United States
}

ABSTRACT: The stochastic formulation of multiexciton generation (MEG) rates is extended to provide access to MEG efficiencies in nanostructures containing thousands of atoms. The formalism is applied to a series of $\mathrm{CdSe} / \mathrm{CdS}$ seeded nanorod heterostructures with different core and shell dimensions. At energies above $3 E_{\mathrm{g}}$ (where $E_{\mathrm{g}}$ is the band gap), the MEG yield increases with decreasing core size, as expected for spherical nanocrystals. Surprisingly, this behavior is reversed for energies below this value, and is explained by the dependence of the density of states near the valence band edge, which increases with the core diameter. Our predictions indicate that the onset of MEG can be shifted to lower energies by manipulating the density of states in complex nanostructure geometries.

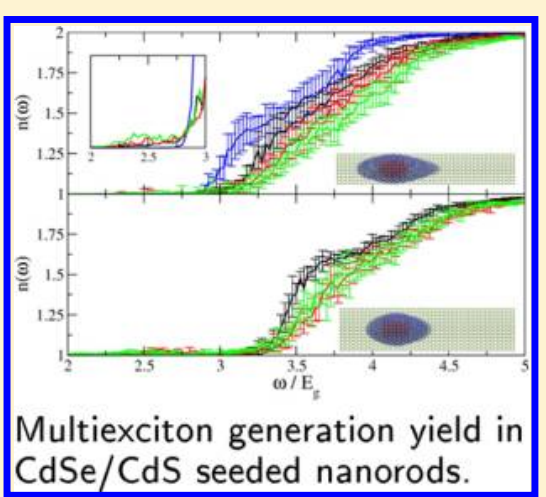

SECTION: Physical Processes in Nanomaterials and Nanostructures

$\mathrm{M}$ ultiexciton generation by which more than a single electron-hole pair is generated from a single photon upon optical excitation is a promising direction to collect "hot" carriers, potentially increasing photovoltaic power conversion efficiencies in solar cells to above $45 \%{ }^{1,2}$ It is common to define an onset photon energy $E_{\text {on }}$ beyond which multiexciton generation (MEG) efficiencies are noticeable. One of the necessary conditions for MEG to be of technological use is that $E_{\text {on }}$ be close to the lower bound of twice the quasiparticle band gap $\left(2 E_{\mathrm{g}}\right){ }^{1}$ In bulk semiconductors, $E_{\mathrm{on}} / E_{\mathrm{g}}>5$, and thus, MEG is not useful for photovoltaic applications. This large ratio is a consequence of energy and momentum conservation constraints, which severely limit the number of multiexciton states that can be formed, coupled with the existence of very rapid nonradiative relaxation pathways. ${ }^{3,4}$ Confined semiconductor nanocrystals (NCs), on the other hand, offer improved MEG efficiencies, ${ }^{5,6}$ with an onset energy below $E_{\text {on }}<3 E_{\mathrm{g}}{ }^{7-17}$ This result was rationalized by several different theoretical treatments. ${ }^{18-31}$ Perhaps the most intuitively appealing picture of MEG in NCs is that of impact ionization. ${ }^{18,19,21,29}$ Using this framework and a unified Green function approach to MEG, it was shown that size scaling of the band gap, Coulomb couplings, and density of states (DOS) lead to an overall increase in MEG rates and efficiencies with decreasing NC size when the photon energy is scaled by $E_{\mathrm{g}}{ }^{32}$

Several different approaches have been proposed recently to lower the onset energy ratio $E_{\mathrm{on}} / E_{\mathrm{g}}$. Gabor et al. ${ }^{33}$ used internal fields to decrease $E_{\mathrm{on}} / E_{\mathrm{g}}$ in carbon nanotube photodiodes, a result that was rationalized by field-induced acceleration of the charge carriers. ${ }^{34}$ Sandberg et al. ${ }^{35}$ studied the role of shape on MEG efficiency and argued for a $60 \%$ increase in MEG yields in semiconductor nanorods (NRs) compared to spherical NCs.
The effect of NR diameter and length on MEG rates was analyzed by considering the scaling of the density of trion states (DOTS) and the Coulomb couplings. ${ }^{36}$ Both show distinct scaling from spherical NCs, leading to a surprising result where the MEG rates, which are given by the product of the two, were roughly independent of the NR length. ${ }^{36}$ This prediction was recently verified by experiments. 37,38

Two additional handles can be used to control the onset energy ratio $E_{\mathrm{on}} / E_{\mathrm{g}}$. The first is based on suppressing the competing channel of phonon emission. Klimov and coworkers have recently shown that the MEG onset energy in type-II core-shell NCs can be reduced markedly by partially blocking the nonradiative decay channel. ${ }^{39}$ Theoretically, addressing the role of phonon emission in nanostructures is rather challenging. ${ }^{40,41}$ The other handle, which forms the crux of our approach, is based on controlling the DOS near the band edge. This allows for reduction of $E_{\text {on }} / E_{\mathrm{g}}$ and at the same time an increase of the MEG efficiency. In order to achieve this, we study the MEG efficiency in seeded NR heterostructures ${ }^{42,43}$ using a stochastic formulation of the MEG formation rates. ${ }^{30}$ We then extend the formalism to calculate the MEG efficiency in a series of seeded NRs with different seed size and different NR diameter. In contrast to the case of spherical NCs, we find that below $E_{\text {on }} / E_{\mathrm{g}}$, the MEG efficiency increases with the seed radius, that is, larger seeds show higher MEG efficiency even when the photon energy is scaled by $E_{\mathrm{g}}$.

In order to calculate the MEG efficiencies in seeded NRs, we adopt the master equation approach, which assumes that each

Received: May 22, 2014

Accepted: July 5, 2014 
exciton generated upon absorption can either decay to the band edge by phonon emission with a rate $\gamma$ or produce another electron-hole pair with a rate $\Gamma_{S}$. The master equation approach was previously applied to study MEG in spherical NCs and predicts MEG efficiencies similar to those calculated by a direct absorption Green function formalism within the wide band limit. ${ }^{32}$ Using the master equation approach, it is straightforward to show that the MEG efficiency is related to the number of excitons generated at steady state, which is given by $^{21}$

$$
n_{\mathrm{ex}}(\omega)=\sum_{\mathrm{S}} P_{\mathrm{S}}(\omega) \frac{2 \Gamma_{\mathrm{S}}+\gamma}{\Gamma_{\mathrm{S}}+\gamma}
$$

where $n_{\mathrm{ex}}(\omega)$ represents the number of excitons generated at a photon energy $\hbar \omega$ (the MEG efficiency is simply given by $\left.n_{\mathrm{ex}}(\omega)-1\right)$ and $P_{\mathrm{S}}(\omega)$ is the probability for absorbing the photon and generating an exciton $|S\rangle$ with energy $E_{S}$

$$
P_{S}(\omega)=\frac{\left|\mu_{0 S}\right|^{2} \delta\left(E_{S}-\hbar \omega\right)}{\sum_{S^{\prime}}\left|\mu_{0 S^{\prime}}\right|^{2} \delta\left(E_{S^{\prime}}-\hbar \omega\right)}
$$

In the above equations, $|S\rangle \equiv\left|S_{i \sigma}^{a \sigma}\right\rangle=a_{a \sigma}^{\dagger} a_{i \sigma}|0\rangle$ is a shorthand notation for a single exciton state obtained by applying the annihilation $\left(a_{i \sigma}\right)$ and creation $\left(a_{a \sigma}^{\dagger}\right)$ operators on the ground state Slater determinant, $|0\rangle$. We use indices $i, j, k, \ldots$ for occupied (hole) states and $a, b, c, \ldots$ for unoccupied (electron) states. $r, s, t, u, \ldots$ are general indices, and $\sigma=\uparrow, \downarrow$ is the spin index. $\mu_{0 S}$ is the transition dipole from $|0\rangle$ to $|S\rangle, \gamma$ is the phonon emission rate, and $\Gamma_{\mathrm{S}}$ is the MEG rate from state $|S\rangle$.

It is interesting to note two limits of eq 1: when the MEG rate is much larger than the phonon emission rate $\left(\Gamma_{S} \gg \gamma\right)$, MEG becomes highly efficient $\left(n_{\mathrm{ex}}(\omega) \rightarrow 2\right)$, and when the phonon emission rate is much larger compared to the MEG rate $\left(\gamma \gg \Gamma_{\mathrm{S}}\right)$, MEG is suppressed $\left(n_{\mathrm{ex}}(\omega) \rightarrow 1\right)$.

The calculation of $\gamma$ and $\Gamma_{\mathrm{S}}$ is computationally expensive for large NCs. We take the value of $\gamma$ from experiments and assume that it is energy-independent. ${ }^{32} \Gamma_{S}$ is written as as sum of rates of creating positive and negative trions

$$
\Gamma_{\mathrm{S}} \equiv \Gamma_{i a}=\Gamma_{a}^{-}+\Gamma_{i}^{+}
$$

and a stochastic formula for the negative and positive trion formation rates is adopted ${ }^{30}$

$$
\begin{aligned}
& \Gamma_{a}^{-}=\frac{2 \pi}{\hbar}\left\langle W_{a}^{2}\right\rangle \rho_{\mathrm{T}}^{-}\left(\varepsilon_{a}\right) \\
& \Gamma_{i}^{+}=\frac{2 \pi}{\hbar}\left\langle W_{i}^{2}\right\rangle \rho_{\mathrm{T}}^{+}\left(\varepsilon_{i}\right)
\end{aligned}
$$

where the negative and positive DOTS is given by

$$
\begin{aligned}
& \rho_{\mathrm{T}}^{-}(\varepsilon)=\sum_{b c j} \delta\left(\varepsilon-\left(\varepsilon_{b}+\varepsilon_{c}-\varepsilon_{j}\right)\right) \\
& \rho_{\mathrm{T}}^{+}(\varepsilon)=\sum_{j k b} \delta\left(\varepsilon-\left(\varepsilon_{j}+\varepsilon_{k}-\varepsilon_{b}\right)\right)
\end{aligned}
$$

and the average square Coulomb coupling for electrons and holes is given by

$$
\begin{aligned}
\left\langle W_{a}^{2}\right\rangle & =\frac{\sum_{b c j} W_{a ; c b j}^{2} \delta\left(\varepsilon_{a}-\left(\varepsilon_{b}+\varepsilon_{c}-\varepsilon_{j}\right)\right)}{\rho_{\mathrm{T}}^{-}\left(\varepsilon_{a}\right)} \\
\left\langle W_{i}^{2}\right\rangle & =\frac{\sum_{j k b} W_{i ; j k b}^{2} \delta\left(\varepsilon-\left(\varepsilon_{j}+\varepsilon_{k}-\varepsilon_{b}\right)\right)}{\rho_{\mathrm{T}}^{+}\left(\varepsilon_{i}\right)}
\end{aligned}
$$

In the above equations, the squared Coulomb couplings are given by

$$
\begin{aligned}
& W_{a ; c b j}^{2}=2\left|V_{a c j b}-V_{j c a b}\right|^{2}+\left|V_{j c a b}\right|^{2}+\left|V_{a c j b}\right|^{2} \\
& W_{i ; j k b}^{2}=2\left|V_{j i b k}-V_{k i j b}\right|^{2}+\left|V_{k i j b}\right|^{2}+\left|V_{j i b k}\right|^{2}
\end{aligned}
$$

where

$$
V_{r s u t}=\iint \mathrm{d}^{3} r \mathrm{~d}^{3} r^{\prime} \frac{\psi_{r}(\mathbf{r}) \psi_{s}(\mathbf{r}) \psi_{u}\left(\mathbf{r}^{\prime}\right) \psi_{t}\left(\mathbf{r}^{\prime}\right)}{\epsilon\left|\mathbf{r}-\mathbf{r}^{\prime}\right|}
$$

is the screened Coulomb interactions and $\psi_{r}(\mathbf{r})$ are the singleparticle orbitals (see more details below). We assume a static dielectric screening, $\epsilon$, for the Coulomb matrix elements.

To calculate the MEG efficiency given by eq 1 , one requires as input the MEG rates and the transition probabilities for all possible single excitons $|S\rangle \equiv\left|S_{i \sigma}^{a \sigma}\right\rangle$. For spherical NCs, these are readily available for moderate excitation energies $\left(\leq 3 E_{\mathrm{g}}\right)^{21}$ However, for NRs (and also seeded NRs), it is practically impossible to generate all possible singly excited states, even at relatively low excitation energies, due to the large DOS. ${ }^{36}$ Therefore, in order to obtain the MEG efficiency, we propose a stochastic approach, where we sample the singly excited states and the corresponding MEG rates rather than calculating them all to perform the full summation formula for $n_{\mathrm{ex}}(\omega)$. The approach can be summarized as follows:

(1) Calculate the single-particle DOS $(\rho(\varepsilon))$ using a stochastic trace formula. ${ }^{30}$ Use convolutions to generate the density of single excited states $\left(\rho_{\mathrm{X}}(E)\right)$ and the negative/ positive trion DOS $\left(\rho_{\mathrm{T}}^{-}(\varepsilon) / \rho_{\mathrm{T}}^{+}(\varepsilon)\right)$. $^{30}$

(2) Generate the negative/positive trion formation rates (eq 4) using the stochastic procedure. ${ }^{30}$

(3) Filter target electron and hole states with energies sampled from $\rho(\varepsilon)$ (above and below the Fermi energy, respectively), and for each electron-hole pair, calculate the transition dipole $\mu_{0 \mathrm{~S}}$ at energy $E_{\mathrm{S}}$.

(4) Use $\Gamma_{a}^{-}$and $\Gamma_{i}^{+}$to generate the MEG rate for each electron-hole pair: $\Gamma_{\mathrm{S} \equiv i a}=\Gamma_{a}^{-}+\Gamma_{i}^{+}$. Obtain $n_{\mathrm{ex}}(\omega)$ by replacing the full summation expression (cf., eq 1 ) with an average over the sampled electron-hole pairs: $n_{\mathrm{ex}}(\omega)=\left(1 / N_{\mathrm{S}}\right) \sum_{\mathrm{S}} \mathcal{P}_{\mathrm{S}}(\omega)$ $\left[\left(2 \Gamma_{\mathrm{S}}+\gamma\right) /\left(\Gamma_{\mathrm{S}}+\gamma\right)\right]$, where $\mathcal{P}_{\mathrm{S}}(\omega)=\rho\left(\varepsilon_{a}\right) \rho\left(\varepsilon_{i}\right) P_{\mathrm{S}}(\omega)$. The product $\rho\left(\varepsilon_{a}\right) \rho\left(\varepsilon_{i}\right)$ is used to correct for the density of electron and hole pairs at energy $E_{\mathrm{S}} . N_{\mathrm{S}}$ is the number of excited states sampled at energy $E_{S}=\hbar \omega$, typically taken to be $N_{S}=200$.

The first two steps have been developed previously by Baer and Rabani and applied to both NCs and NRs. ${ }^{30}$ The last two provide an extension to calculate the MEG efficiencies by sampling the singly excited states rather than calculating them all.

In Figure 1, we plot the negative and positive trion formations rates (upper panel, shown also in ref 30) and the MEG efficiency (lower panel, shown here for the first time) for a $3 \mathrm{~nm}$ diameter $\mathrm{CdSe}$ NC. The stochastic results are compared with the full summation approach, labeled "deterministic". The agreement between the stochastic and deterministic calculations is excellent, although the stochastic rates are slightly 


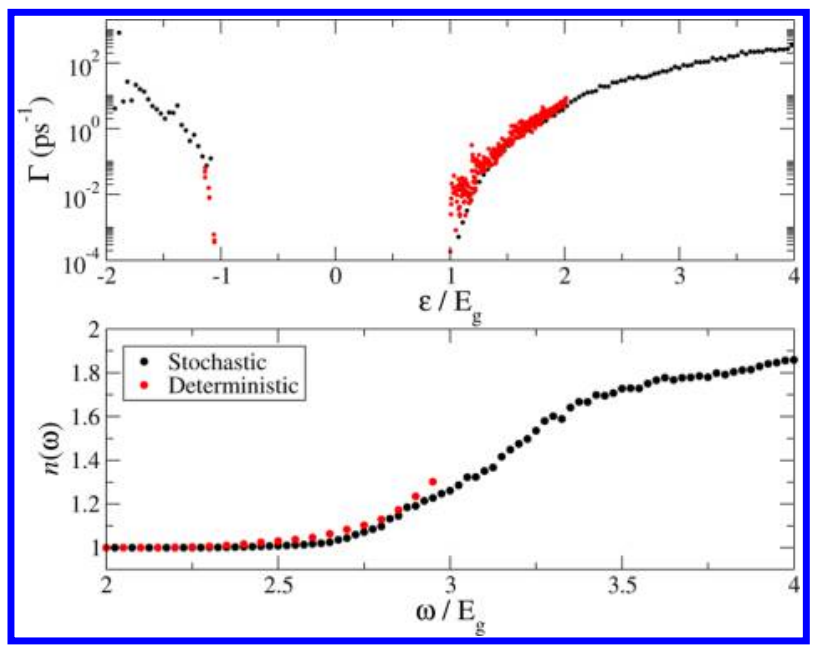

Figure 1. Trion formation rate (upper panel) and the MEG efficiency (lower panel) for a $3 \mathrm{~nm}$ diameter $\mathrm{CdSe} \mathrm{NC}\left(\mathrm{Cd}_{232} \mathrm{Se}_{251}\right)$ as a function of scaled energy ( $\omega$ is the photon energy, while $\varepsilon$ is the energy of the electron (hole), for negative (positive) trions, measured from the bottom of the conduction (top of the valence) band). The full summation approach labeled "deterministic" is shown by red circles, and the stochastic approach spanning a larger energy range is shown by black circles.

underestimated compared with the average deterministic rates. ${ }^{30}$ Comparing the results near the valence and conduction band edges, it is clear that the uniform sampling approach of the energy falls short in describing the trion formation rates for $\varepsilon / E_{\mathrm{g}} \rightarrow \pm 1$. However, because these rates are very low compared to the phonon emission rate, $\gamma$, assuming a vanishing value does not affect the MEG efficiencies.

In the lower panel of Figure 1, we compare the stochastic MEG efficiencies to the deterministic approach. The stochastic approach slightly underestimates $n_{\mathrm{ex}}(\omega)$, resulting from the small systematic deviation of the trion formation rates. However, the overall agreement is excellent, and the stochastic approach is reliable at energies much higher than those accessible by the deterministic calculations.

Moving on to the CdSe/CdS core/shell NRs of $20 \mathrm{~nm}$ length, we calculated the MEG formation rates and efficiencies for rod diameters $D_{\text {rod }}$ of 4 and $6 \mathrm{~nm}$ and for several core diameters $D_{\text {core }}$ in the range of $2-4.5 \mathrm{~nm}$ in each case. The rods were faceted, and the center of the spherical CdSe core was placed at $1 / 3$ of the length of the rod. The configurations of the seeded rods were relaxed using molecular dynamics runs for $100 \mathrm{ps}$ at $300 \mathrm{~K}$ with a recently developed force field ${ }^{44}$ accurately describing $\mathrm{CdSe} / \mathrm{CdS}$ heterostructures. ${ }^{45}$ The final configurations were quenched to remove structural effects of thermal fluctuations; thus, only one trajectory was used for each structure. The single-particle orbitals $\left(\psi_{r}(\mathbf{r})\right)$ required for the calculation of the MEG rates were then generated for the relaxed configurations using semiempirical pseudopotential models on a real space grid, ${ }^{46,47}$ with a Gaussian filtering function. $^{48}$

In Figure 2, we show the MEG efficiencies (left panels) and the trion formation rates (right panels) for the two sets of rod diameters. The MEG efficiency increases with photon energy with an onset close to $3 E_{\mathrm{g}}$ and $3.5 E_{\mathrm{g}}$ for the $D_{\text {rod }}=4$ and $6 \mathrm{~nm}$, respectively. This is consistent with experiments for a wide range of confined nanostructures. ${ }^{5}$ At energies above the onset energy, we find that the MEG efficiency at a scaled energy increases as the size of the NC seed decreases, consistent with calculations of spherical NCs. ${ }^{21}$ For photon energies below the onset (between $2 E_{\mathrm{g}}$ and $3 E_{\mathrm{g}}$ ), the reverse is true, and the MEG efficiency is higher for larger seeds. This is depicted in the inset of Figure 2 and is qualitatively different from spherical NCs.

Despite the fact that the MEG efficiencies for energies between $2 E_{\mathrm{g}}$ and $3 E_{\mathrm{g}}$ are rather small in seeded NRs, understanding the origin of the behavior below the onset is still significant as it may prove useful in designing materials with higher MEG in this important range of energies. In the right panels of Figure 2, we plot the negative trion formation rate $\left(\Gamma_{e}^{-}(\varepsilon)\right)$ for all corresponding cases. Because the MEG process is dominated by the formation of negative trions for CdSe $\mathrm{NCs},{ }^{21}$ we focus on this channel and ignore, for the sake of the discussion (not in the calculations of the MEG efficiencies), the positive channel. For all cases studied, $\Gamma_{e}^{-}(\varepsilon)$ increases with the electron energy, similar to the behavior of spherical ${ }^{29,32}$ and rod-shaped ${ }^{36}$ nanostructures. However, there seems to be an isosbestic point about $\varepsilon / E_{\mathrm{g}}=1.75$ where $\Gamma_{e}^{-}(\varepsilon)$ changes behavior (this is more clearly observed in the DOTS discussed below). This isosbestic point is correlated with the crossover of the MEG efficiency with the NC seed size.

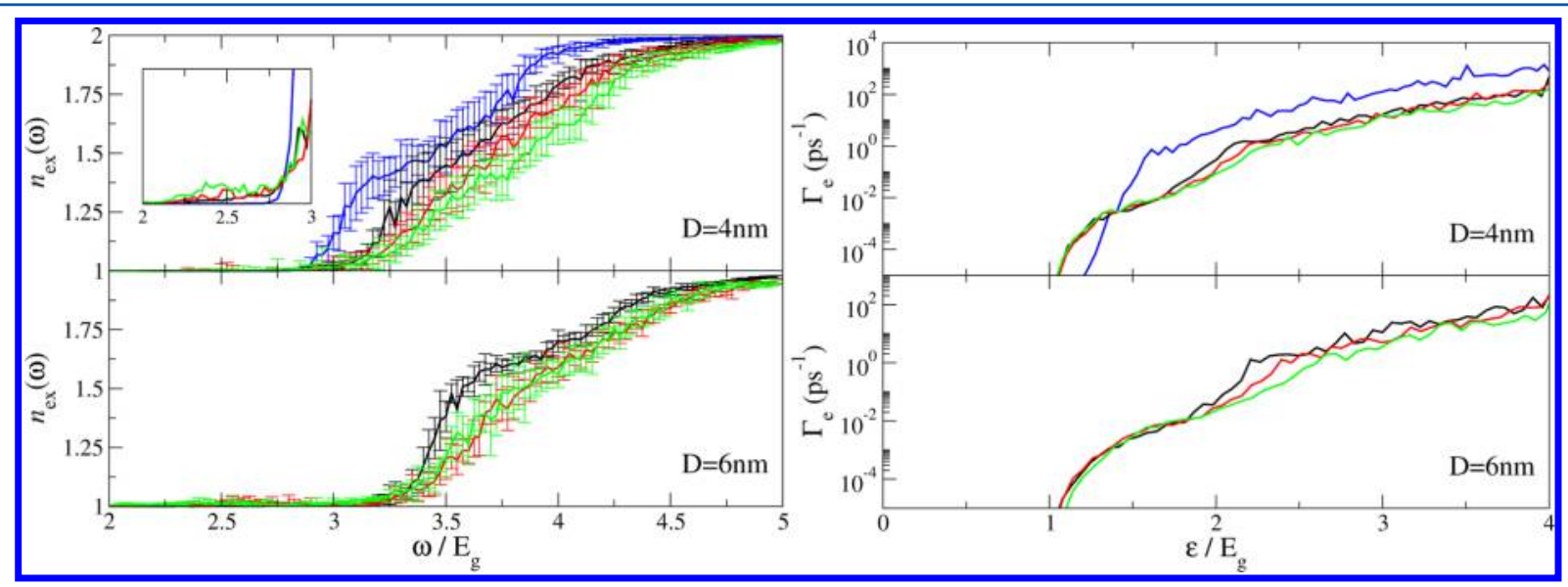

Figure 2. MEG efficiency for $D_{\text {rod }}=4$ and $6 \mathrm{~nm}$ diameter NRs (upper and lower left panels, respectively) and the corresponding negative trion formation rates (right panels) for scaled energies. For the $D_{\text {rod }}=4 \mathrm{~nm}$ rods, the blue, black, red, and green curves represent seed sizes of $D_{\text {core }}=0$, 2 , 2.5 , and $3 \mathrm{~nm}$, respectively. For the $D_{\text {rod }}=6 \mathrm{~nm}$ rod, the black, red, and green curves represent seed sizes of $D_{\text {core }}=2.5,3.5$, and $4.5 \mathrm{~nm}$, respectively. (Inset) Zoom in on the low-energy range. 


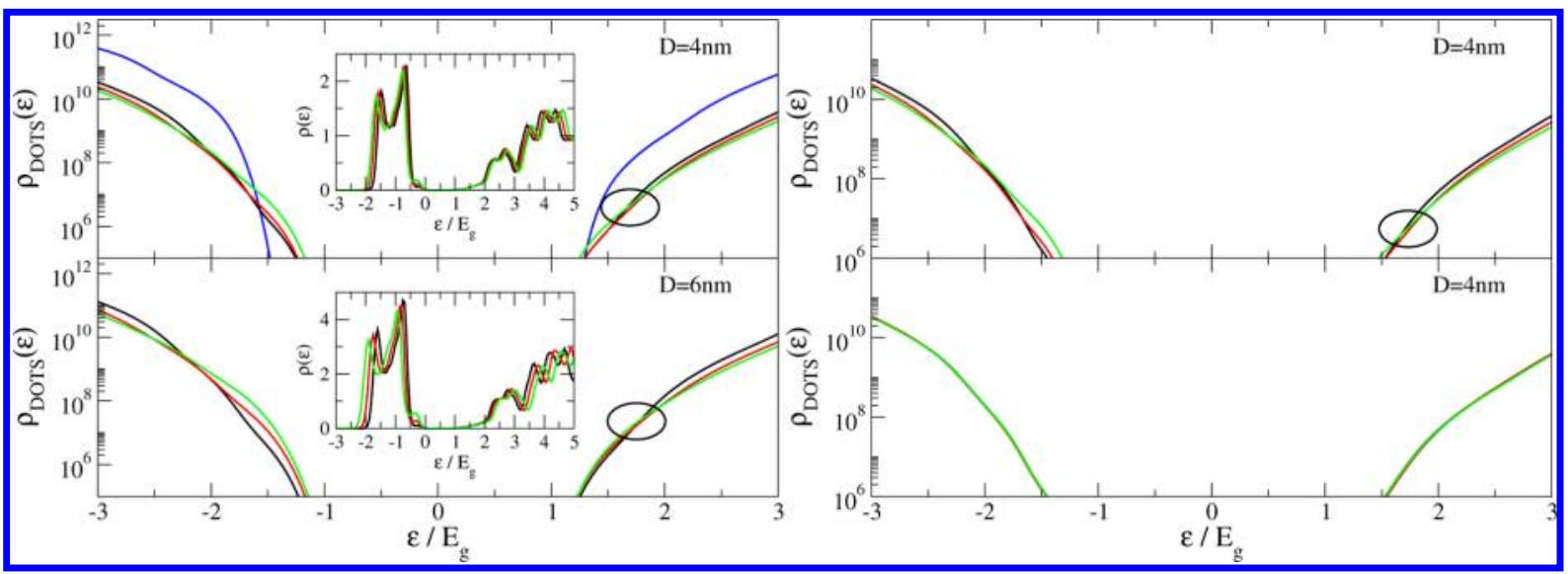

Figure 3. DOTS for $D_{\text {rod }}=4$ and $6 \mathrm{~nm}$ NRs (upper and lower panels, respectively) and the artificial DOTS for a $D_{\text {rod }}=4 \mathrm{~nm}$ diameter (right panels) as described in the text. Insets show the corresponding DOS for each $D_{\text {rod }}$. For the $D_{\text {rod }}=4 \mathrm{~nm}$ rod, the blue, black, red, and green curves represent seed sizes of $D_{\text {core }}=0,2,2.5$, and $3 \mathrm{~nm}$, respectively. For the $6 \mathrm{~nm}$ rod, the black, red, and green curves represent seed sizes of $D_{\text {core }}=2.5$, 3.5, and $4.5 \mathrm{~nm}$, respectively.

To delineate the factors governing this behavior, we first note that the trion formation rates are given in terms of a product of the average square Coulomb coupling and the DOTS (cf., eq 4). The former shows a monotonic core-size dependence and a weak energy dependence (not shown). ${ }^{30,36}$ Therefore, the isosbestic point must reflect a nonmonotonic behavior of the DOTS with $D_{\text {core }}$ which is indeed the case, as shown by the left panels of Figure 3. We find that both $\rho_{\mathrm{T}}^{-}(\varepsilon)$ and $\rho_{\mathrm{T}}^{+}(\varepsilon)$ exhibit an isosbestic point, while that for the negative DOTS (circled in black) is correlated with the isosbestic point of $\Gamma_{e}^{-}(\varepsilon)$.

Because the DOTS is given in terms of a triple convolution of the DOS, we also plot the DOS as insets to each panel in Figure 3. We focus on the energy regime about the Fermi energy (which is assumed at the top of the valence band and is set as the origin of the energy axis). Perhaps the most notable effect of the seed size on the DOS is the appearance of a shoulder near the valence band edge as $D_{\text {core }}$ increases. This shoulder reflects the increasing DOS on the seed due to volume effects. In addition, the DOS shows a systematic shift in the position of the valence and conduction peaks resulting from small changes in the band gap as $D_{\text {core }}$ increases (note that we scale the energy by the band gap $E_{\mathrm{g}}$ for each system). Here, the decrease of the band gap was mainly attributed to the change of the highest hole energy, which is localized on the seed. ${ }^{47}$

In the right panels of Figure 3, we plot the DOTS for two additional cases. The bottom panel assumes that the DOS of the holes for all seed sizes is identical; therefore, the observed change in the DOTS reflects the differences in the DOS of the electrons (i.e., the portion of the DOS above the Fermi energy). Clearly, all three sizes give the same DOTS. Therefore, one can conclude that the isosbestic point observed in the negative DOTS originates from the hole DOS (i.e., the portion of the DOS below the Fermi energy). The upper right panel of Figure 3 shows the DOTS for the case where the only difference in the DOS for the three values of $D_{\text {core }}$ is assumed to be the aforementioned shoulder near the valence band edge. Clearly, this is sufficient to account for the isosbestic point. Thus, we conclude that the crossover behavior observed in the MEG efficiency can be attributed to the increase in the DOS near the valence band edge and at the same time the rather insignificant decrease in the band gap energy.

In conclusion, we have extended our stochastic approach to calculate the MEG efficiency in systems containing thousands of atoms within a master equation formalism. Rather than calculating all possible excitations and the corresponding MEG rates, we proposed to sample the electron-hole pairs and, for each pair, obtain the MEG rates (sum of the positive and negative trion formation rates) using our recently developed expeditious stochastic approach. ${ }^{30}$ Application of the approach to quasi-type-II $\mathrm{CdSe} / \mathrm{CdS}$ seeded NRs uncovered an interesting and unexpected behavior. Above the onset of MEG (around $3 E_{\mathrm{g}}$ ), the efficiency increases as $D_{\text {core }}$ decreases, as expected. However, below this onset, the opposite is true, and larger seeds show higher MEG efficiencies, contrary to the situation in spherical NCs. This anomalous behavior was caused by the marked increase in DOS near the valence band edge as $D_{\text {core }}$ dropped and the relative insensitivity of $E_{\mathrm{g}}$ to $D_{\text {core }}$ (due to the quasi-type-II band alignment) .

Manipulating the DOS without affecting the band gap can be used to control MEG efficiencies in other semiconducting heterostructures. Of particular relevance are type-II NRs, ${ }^{49}$ which are expected to yield larger MEG efficiencies, perhaps also due to the large internal fields across the interface between the two semiconducting materials. ${ }^{33,34}$ These and other possible scenarios are currently under investigation.

\section{AUTHOR INFORMATION}

\section{Corresponding Authors}

*E-mail: hagai.eshet@gmail.com (H.E.)

*E-mail: roi.baer@huji.ac.il (R.B.)

*E-mail: dxn@chem.ucla.edu (D.N.)

*E-mail: eran.rabani@gmail.com (E.R.)

Notes

The authors declare no competing financial interest.

\section{ACKNOWLEDGMENTS}

R.B. and E.R. gratefully thank the Israel Science Foundation, Grants 1020/10 and No. 611/11, respectively. R.B. and D.N. acknowledge the support of the U.S.-Israel Bi-National Science Foundation. E.R. would like to thank the Center for ReDefining Photovoltaic Efficiency through Molecule Scale Control, an Energy Frontier Research Center funded by the U.S. Department of Energy, Office of Science, Office of Basic Energy Sciences under Award Number DE-SC0001085. 


\section{REFERENCES}

(1) Beard, M. C.; Midgett, A. G.; Hanna, M. C.; Luther, J. M.; Hughes, B. K.; Nozik, A. J. Comparing Multiple Exciton Generation in Quantum Dots to Impact Ionization in Bulk Semiconductors: Implications for Enhancement of Solar Energy Conversion. Nano Lett. 2010, 10, 3019-3027.

(2) Klimov, V. I. Detailed-Balance Power Conversion Limits of Nanocrystal-Quantum-Dot Solar Cells in the Presence of Carrier Multiplication. Appl. Phys. Lett. 2006, 89, 123118.

(3) Christensen, O. Quantum Efficiency of Internal Photoelectric Effect in Silicon and Germanium. J. Appl. Phys. 1976, 47, 689-695.

(4) Kolodinski, S.; Werner, J. H.; Wittchen, T.; Queisser, H. J. Quantum Efficiencies Exceeding Unity Due to Impact Ionization in Silicon Solar-Cells. Appl. Phys. Lett. 1993, 63, 2405-2407.

(5) Padilha, L. A.; Stewart, J. T.; Sandberg, R. L.; Bae, W. K.; Koh, W.-K.; Pietryga, J. M.; Klimov, V. I. Carrier Multiplication in Semiconductor Nanocrystals: Influence of Size, Shape, and Composition. Acc. Chem. Res. 2013, 46, 1261-1269.

(6) Shabaev, A.; Hellberg, C. S.; Efros, A. L. Efficiency of Multiexciton Generation in Colloidal Nanostructures. Acc. Chem. Res. 2013, 46, 1242-1251.

(7) Schaller, R. D.; Klimov, V. I. High Efficiency Carrier Multiplication in PbSe Nanocrystals: Implications for Solar Energy Conversion. Phys. Rev. Lett. 2004, 92, 186601.

(8) Schaller, R. D.; Agranovich, V. M.; Klimov, V. I. High-Efficiency Carrier Multiplication through Direct Photogeneration of MultiExcitons via Virtual Single-Exciton States. Nat. Phys. 2005, 1, 189194.

(9) Ellingson, R. J.; Beard, M. C.; Johnson, J. C.; Yu, P. R.; Micic, O. I.; Nozik, A. J.; Shabaev, A.; Efros, A. L. Highly Efficient Multiple Exciton Generation in Colloidal PbSe and PbS Quantum Dots. Nano Lett. 2005, 5, 865-871.

(10) Schaller, R. D.; Petruska, M. A.; Klimov, V. I. Effect of Electronic Structure on Carrier Multiplication Efficiency: Comparative Study of PbSe and CdSe Nanocrystals. Appl. Phys. Lett. 2005, 87, 253102.

(11) Murphy, J. E.; Beard, M. C.; Norman, A. G.; Ahrenkiel, S. P.; Johnson, J. C.; Yu, P. R.; Micic, O. I.; Ellingson, R. J.; Nozik, A. J. PbTe Colloidal Nanocrystals: Synthesis, Characterization, and Multiple Exciton Generation. J. Am. Chem. Soc. 2006, 128, 3241-3247.

(12) Schaller, R. D.; Sykora, M.; Jeong, S.; Klimov, V. I. HighEfficiency Carrier Multiplication and Ultrafast Charge Separation in Semiconductor Nanocrystals Studied via Time-Resolved Photoluminescence. J. Phys. Chem. B 2006, 110, 25332-25338.

(13) Schaller, R. D.; Sykora, M.; Pietryga, J. M.; Klimov, V. I. Seven Excitons at a Cost of One: Redefining the Limits for Conversion Efficiency of Photons into Charge Carriers. Nano Lett. 2006, 6, 424429.

(14) Beard, M. C.; Knutsen, K. P.; Yu, P. R.; Luther, J. M.; Song, Q.; Metzger, W. K.; Ellingson, R. J.; Nozik, A. J. Multiple Exciton Generation in Colloidal Silicon Nanocrystals. Nano Lett. 2007, 7, $2506-2512$.

(15) Schaller, R. D.; Pietryga, J. M.; Klimov, V. I. Carrier Multiplication in InAs Nanocrystal Quantum Dots with an Onset Defined by the Energy Conservation Limit. Nano Lett. 2007, 7, 34693476.

(16) Trinh, M. T.; Polak, L.; Schins, J. M.; Houtepen, A. J.; Vaxenburg, R.; Maikov, G. I.; Grinbom, G.; Midgett, A. G.; Luther, J. M.; Beard, M. C.; et al. Anomalous Independence of Multiple Exciton Generation on Different Group IV-VI Quantum Dot Architectures. Nano Lett. 2011, 11, 1623-1629.

(17) Stewart, J. T.; Padilha, L. A.; Qazilbash, M. M.; Pietryga, J. M.; Midgett, A. G.; Luther, J. M.; Beard, M. C.; Nozik, A. J.; Klimov, V. I. Comparison of Carrier Multiplication Yields in $\mathrm{PbS}$ and $\mathrm{PbSe}$ Nanocrystals: The Role of Competing Energy-Loss Processes. Nano Lett. 2012, 12, 622-628.

(18) Franceschetti, A.; An, J. M.; Zunger, A. Impact Ionization Can Explain Carrier Multiplication in PbSe Quantum Dots. Nano Lett. 2006, 6, 2191-2195.
(19) Allan, G.; Delerue, C. Role of Impact Ionization in Multiple Exciton Generation in PbSe Nanocrystals. Phys. Rev. B 2006, 73, 205423.

(20) Shabaev, A.; Efros, A. L.; Nozik, A. J. Multiexciton Generation by a Single Photon in Nanocrystals. Nano Lett. 2006, 6, 2856-2863.

(21) Rabani, E.; Baer, R. Distribution of Multiexciton Generation Rates in CdSe and InAs Nanocrystals. Nano Lett. 2008, 8, 4488-4492.

(22) Isborn, C. M.; Kilina, S. V.; Li, X. S.; Prezhdo, O. V. Generation of Multiple Excitons in PbSe and CdSe Quantum Dots by Direct Photoexcitation: First-Principles Calculations on Small PbSe and CdSe Clusters. J. Phys. Chem. C 2008, 112, 18291-18294.

(23) Isborn, C. M.; Prezhdo, O. V. Charging Quenches Multiple Exciton Generation in Semiconductor Nanocrystals: First-Principles Calculations on Small PbSe Clusterse. J. Phys. Chem. C 2009, 113, 12617-12621.

(24) Delerue, C.; Allan, G.; Pijpers, J. J. H.; Bonn, M. Carrier Multiplication in Bulk and Nanocrystalline Semiconductors: Mechanism, Efficiency, and Interest for Solar Cells. Phys. Rev. B 2010, 81, 125306.

(25) Witzel, W. M.; Shabaev, A.; Hellberg, C. S.; Jacobs, V. L.; Efros, A. L. Quantum Simulation of Multiple-Exciton Generation in a Nanocrystal by a Single Photon. Phys. Rev. Lett. 2010, 105, 137401.

(26) Fischer, S. A.; Madrid, A. B.; Isborn, C. M.; Prezhdo, O. V. Multiple Exciton Generation in Small Si Clusters: A High-Level, Ab Initio Study. J. Phys. Chem. Lett. 2010, 1, 232-237.

(27) Piryatinski, A.; Velizhanin, K. A. An Exciton Scattering Model for Carrier Multiplication in Semiconductor Nanocrystals: Theory. J. Chem. Phys. 2010, 133, 084508.

(28) Allan, G.; Delerue, C. Optimization of Carrier Multiplication for More Effcient Solar Cells: The Case of Sn Quantum Dots. ACS Nano 2011, 5, 7318-7323.

(29) Lin, Z.; Franceschetti, A.; Lusk, M. T. Size Dependence of the Multiple Exciton Generation Rate in CdSe Quantum Dots. ACS Nano 2011, 5, 2503-2511.

(30) Baer, R; Rabani, E. Expeditious Stochastic Calculation of Multiexciton Generation Rates in Semiconductor Nanocrystals. Nano Lett. 2012, 12, 2123-2128.

(31) Velizhanin, K. A.; Piryatinski, A. Numerical Analysis of Carrier Multiplication Mechanisms in Nanocrystalline and Bulk Forms of PbSe and PbS. Phys. Rev. B 2012, 86, 165319.

(32) Rabani, E.; Baer, R. Theory of Multiexciton Generation in Semiconductor Nanocrystals. Chem. Phys. Lett. 2010, 496, 227-235.

(33) Gabor, N. M.; Zhong, Z. H.; Bosnick, K.; Park, J.; McEuen, P. L. Extremely Efficient Multiple Electron-Hole Pair Generation in Carbon Nanotube Photodiodes. Science 2009, 325, 1367-1371.

(34) Baer, R.; Rabani, E. Can Impact Excitation Explain Efficient Carrier Multiplication in Carbon Nanotube Photodiodes? Nano Lett. 2010, 10, 3277-3282.

(35) Sandberg, R. L.; Padilha, L. A.; Qazilbash, M. M.; Bae, W. K.; Schaller, R. D.; Pietryga, J. M.; Stevens, M. J.; Baek, B.; Nam, S. W.; Klimov, V. I. Multiexciton Dynamics in Infrared-Emitting Colloidal Nanostructures Probed by a Superconducting Nanowire SinglePhoton Detector. ACS Nano 2012, 6, 9532-9540.

(36) Baer, R.; Rabani, E. Communication: Biexciton Generation Rates in CdSe Nanorods Are Length Independent. J. Chem. Phys. 2013, 138, 051102 .

(37) Padilha, L. A.; Stewart, J. T.; Sandberg, R. L.; Bae, W. K.; Koh, W.-K.; Pietryga, J. M.; Klimov, V. I. Aspect Ratio Dependence of Auger Recombination and Carrier Multiplication in PbSe Nanorods. Nano Lett. 2013, 13, 1092-1099.

(38) Karki, K. J.; Ma, F.; Zheng, K.; Zidek, K.; Mousa, A.; Abdellah, M. A.; Messing, M. E.; Wallenberg, L. R.; Yartsev, A.; Pullerits, T. Multiple Exciton Generation in Nanocrystals Revisited: Consistent Calculation of the Yield Based on Pump-Probe Spectroscopy. Sci. Rep. 2013, 3, 2287.

(39) Klimov, V. I. Multicarrier Interactions in Semiconductor Nanocrystals in Relation to the Phenomena of Auger Recombination and Carrier Multiplication. Annu. Rev. Condens. Mater. Phys. 2014, 5, 285-316. 
(40) Prezhdo, O. V. Multiple Excitons and the Electron-Phonon Bottleneck in Semiconductor Quantum Dots: An Ab Initio Perspective. Chem. Phys. Lett. 2008, 460, 1-9.

(41) Chen, L. L.; Bao, H.; Tan, T. Z.; Prezhdo, O. V.; Ruan, X. L. Shape and Temperature Dependence of Hot Carrier Relaxation Dynamics in Spherical and Elongated CdSe Quantum Dots. J. Phys. Chem. C 2011, 115, 11400-11406.

(42) Carbone, L.; Nobile, C.; De Giorgi, M.; Sala, F. D.; Morello, G.; Pompa, P.; Hytch, M.; Snoeck, E.; Fiore, A.; Franchini, I. R.; et al. Synthesis and Micrometer-Scale Assembly of Colloidal CdSe/CdS Nanorods Prepared by a Seeded Growth Approach. Nano Lett. 2007, 7, 2942-2950.

(43) Li, H.; Kanaras, A. G.; Manna, L. Colloidal Branched Semiconductor Nanocrystals: State of the Art and Perspectives. Acc. Chem. Res. 2013, 46, 1387-1396.

(44) Grünwald, M.; Zayak, A.; Neaton, J. B.; Geissler, P. L.; Rabani, E. Transferable Pair-Potentials for $\mathrm{CdS}$ and $\mathrm{ZnS}$ Crystals. J. Chem. Phys. 2012, 136, 234111.

(45) Grünwald, M.; Lutker, K.; Alivisatos, A. P.; Rabani, E.; Geissler, P. L. Metastability in Pressure-Induced Structural Transformations of CdSe/ZnS Core/Shell Nanocrystals. Nano Lett. 2013, 13, 1367-1372.

(46) Rabani, E.; Hetenyi, B.; Berne, B. J.; Brus, L. E. Electronic Properties of CdSe Nanocrystals in the Absence and Presence of a Dielectric Medium. J. Chem. Phys. 1999, 110, 5355.

(47) Eshet, H.; Grunwald, M.; Rabani, E. The Electronic Structure of CdSe/CdS Core/Shell Seeded Nanorods: Type-I or Quasi-Type-II? Nano Lett. 2013, 13, 5880-5885.

(48) Neuhauser, D. Bound-State Eigenfunctions from Wave-Packets - Time-Energy Resolution. J. Chem. Phys. 1990, 93, 2611-2616.

(49) Luther, J. M.; Zheng, H.; Sadtler, B.; Alivisatos, A. P. Synthesis of $\mathrm{PbS}$ Nanorods and Other Ionic Nanocrystals of Complex Morphology by Sequential Cation Exchange Reactions. J. Am. Chem. Soc. 2009, 131, 16851-16857. 